\title{
Weakly bound water structure, bond valence saturation and water dynamics at the goethite (100) surface/aqueous interface: ab initio dynamical simulations
}

\author{
Ying Chen ${ }^{1}$, Eric J. Bylaska ${ }^{2^{*}}$ (ID and John H. Weare ${ }^{1}$
}

\begin{abstract}
Background: Many important geochemical and biogeochemical reactions occur in the mineral/formation water interface of the highly abundant mineral, goethite $[\mathrm{a}-\mathrm{Fe}(\mathrm{OOH})]$. Ab initio molecular dynamics (AIMD) simulations of the goethite a-FeOOH (100) surface and the structure, water bond formation and dynamics of water molecules in the mineral/aqueous interface are presented. Several exchange correlation functionals were employed (PBE96, PBE96 + Grimme, and PBE0) in the simulations of a $(3 \times 2)$ goethite surface with 65 absorbed water molecules in a 3D-periodic supercell ( $\mathrm{a}=30 \AA$, FeOOH slab $\sim 12 \AA$ thick, solvation layer $\sim 18 \AA$ thick).

Results: The lowest energy goethite (100) surface termination model was determined to have an exposed surface $\mathrm{Fe}^{3+}$ that was loosely capped by a water molecule and a shared hydroxide with a neighboring surface $\mathrm{Fe}^{3+}$. The water molecules capping surface $\mathrm{Fe}^{3+}$ ions were found to be loosely bound at all DFT levels with and without Grimme corrections, indicative that each surface $\mathrm{Fe}^{3+}$ was coordinated with only five neighbors. These long bonds were supported by bond valence theory calculations, which showed that the bond valence of the surface $\mathrm{Fe}^{3+}$ was saturated and surface has a neutral charge. The polarization of the water layer adjacent to the surface was found to be small and affected only the nearest water. Analysis by density difference plots and localized Boys orbitals identified three types of water molecules: those loosely bound to the surface $\mathrm{Fe}^{3+}$, those hydrogen bonded to the surface hydroxyl, and bulk water with tetrahedral coordination. Boys orbital analysis showed that the spin down lone pair orbital of the weakly absorbed water interact more strongly with the spin up $\mathrm{Fe}^{3+}$ ion. These weakly bound surface water molecules were found to rapidly exchange with the second water layer ( $\sim .025$ exchanges/ps) using a dissociative mechanism.
\end{abstract}

Conclusions: Water molecules adjacent to the surface were found to only weakly interact with the surface and as a result were readily able to exchange with the bulk water. To account for the large surface Fe- $\mathrm{OH}_{2}$ distances in the DFT calculations it was proposed that the surface $\mathrm{Fe}^{3+}$ atoms, which already have their bond valence fully satisfied with only five neighbors, are under-coordinated with respect to the bulk coordination.

Keywords: Goethite, Goethite (100) surface, Fe-oxyhydroxide, Mineral water interface, Dissociative exchange, Ab initio molecular dynamics, DFT, Electronic structure, Bond valence theory, Water interaction with mineral surface, Condensed matter Grimme corrections

\footnotetext{
*Correspondence: eric.bylaska@pnnl.gov

${ }^{2}$ William R. Wiley Environmental Molecular Sciences Laboratory, Pacific

Northwest National Laboratory, Richland, WA 99354, USA

Full list of author information is available at the end of the article
} 


\section{Background}

As the thermodynamically most stable Fe-oxyhydroxide, goethite $(\alpha-\mathrm{FeOOH})$ occurs widely in natural environments [1-3] and is the dominant reactive mineral in lake and marine sediments [4]. It is found in weathering products, primary hydrothermal minerals, acid mine drainage precipitates, bog and marine environments [3-5] and has been observed in abundance on Mars [6]. The surface reactivity of the goethite-water interface has been extensively studied and is known to absorb a large number of reactive species including protons $[7,8]$, chromate [9], carboxylates [10], carbonate [11], arsenic [12, 13], and a host of soluble uranium $[14,15]$ and plutonium species [16]. To support interpretation of these processes we report here results of electronic structure simulations of the structure, reactivity and dynamics in the surface/ aqueous liquid interface of this mineral.

The goethite crystal structure has an orthorhombic dipyramidal class symmetry (space group No. 62, Pnma in the Hermann-Mauguin notation [17-19]) as shown in Fig. 1. Rather than the Pnma unit cell, it is common to use the Pbmn unit cell (see Rustad et al. [20] and Randall et al. [21]) with alternative axes specified by the following change of axes (Pnma $\rightarrow$ Pbnm), $x \rightarrow y, y \rightarrow z$, and $z \rightarrow x$. The crystal structure has a perfect cleavage on the (100) ((010) Pbnm) plane. This plane (more clearly illustrated below) is not the main crystal growth surface of this mineral. However it is a common surface and is easily cleaved to provide a well-structured surface for spectroscopic studies $[22,23]$.

Several molecular modeling studies of goethite and the goethite-water interfaces have been reported in the literature [24-28]. Most of these studies have used classical molecular dynamics (MD) to predict the structure and hydration behavior of these interfaces [24-33]. Multisite complexion models (i.e. MUSIC model [34, 35]) have also been used to predict their proton affinity. In addition to molecular dynamics studies, electronic structure calculations, i.e. DFT calculations with no dynamics, have been used to determine the structure and energetics of the goethite surfaces [2, 12, 21, 36], including those with absorbed water [2, 37]. The most thorough of these have been reported by Kubicki et al. [2]. They performed optimization studies for a single water absorbed on the

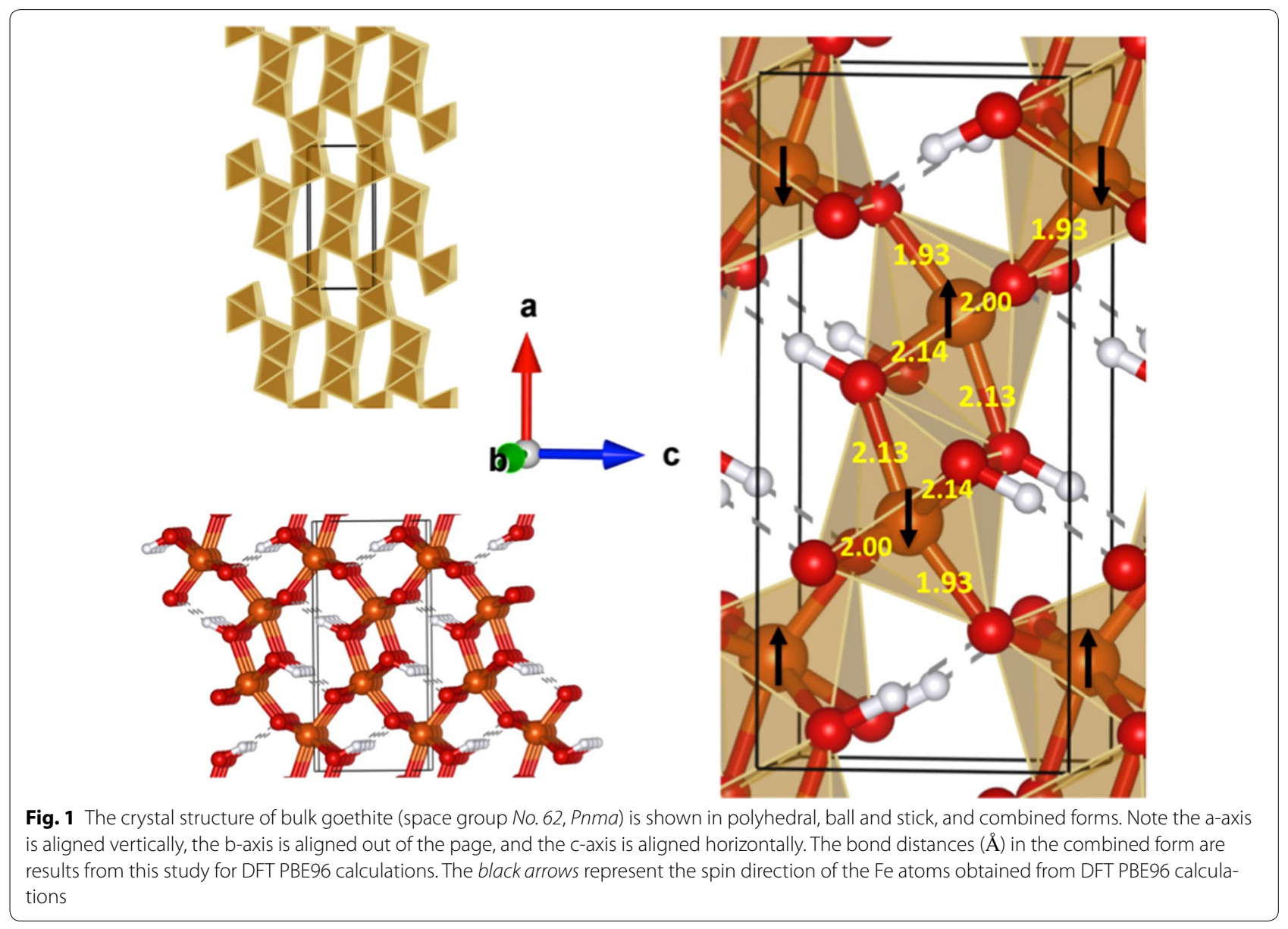


goethite (100) surface termination at the DFT $+\mathrm{U}$ [38] level. In their calculations, the bond length between the first layer of water and the exposed surface $\mathrm{Fe}^{3+}$ atom were found to be very long $(>2.3 \AA)$; the adjacent water only weakly interacting with the surface $\mathrm{Fe}^{3+}$. This suggests that the exposed surface $\mathrm{Fe}^{3+}$ are fivefold coordinated rather than sixfold coordinated as in the bulk [39]. This under-coordination disagrees with prior MD simulations that reported a surface $\mathrm{Fe}^{3+}-\mathrm{OH}_{2}$ distance of $2.13 \AA$ for the isolated surface (2.21 $\AA$ microsolvated) [27] and is does not support the accepted approach for generating surface models for oxides in which the metal cations retain the coordination of the bulk. De Leeuw and Cooper in prior work suggested such an under-coordination was possible for this surface even though their $\mathrm{MD}$ results predicted $\mathrm{Fe}^{3+}-\mathrm{OH}_{2}$ distances that were only slightly longer than bulk $\mathrm{Fe}-\mathrm{OH}$ distances [27].

The results of Kubicki et al. also disagree with recent Crystal Truncation Rod (CTR) studies of the solvated goethite (100) surface by Ghose et al. [22] who obtained a distance of $2.152 \AA$ from the analysis of their CTR intensities. This distance is slightly longer than the $\mathrm{Fe}-\mathrm{OH}$ distances ( 2.09 ̊ experiment, $\sim 2.13 \AA$ DFT) seen for the bulk (see Fig. 1). However, there is some uncertainty in the interpretation of the CTR spectra, because it was fit to a distribution of hypothesized structural models. In their paper, Kubicki et al. [2] rationalized the discrepancies of DFT from prior molecular dynamics results and the CTR experiment by pointing out that the bond length was very sensitive the solvation and additional layers of water molecules were needed to properly model the bulk goethite (100) water interface. Because of the computational cost they were not able to carry out these larger DFT simulations.

For weakly bound systems it is difficult to identify structures in a fluid that reliably reflect the average structure of the fluctuating system for use in direct optimization methods. While dynamics are available from MD, it can be quite challenging to accurately capture the strong polarization and other chemical interactions of the surrounding water molecules near complex transition-metal oxide surfaces [27, 32] using parameterized force fields. $\mathrm{Ab}$ initio molecular dynamics methods (AIMD) implement the calculation of forces on the fly from an approximate solution to the electronic Schrödinger equation (Density Functional Theory, DFT [38, 40, 41]). Chemical changes such as polarization, bond breaking and formation, etc. are straightforwardly incorporated in this approach, but at the cost of a much more expensive time step than MD. In this article we report results using AIMD simulations to predict the structural, bonding and dynamical properties of the solvated ( 6 water layers) goethite (100) surface.

\section{Methods}

All DFT [38] geometry optimizations and Car-Parrinello simulations $[40,41]$ in this study were performed with the plane-wave NWPW module [42] contained in the NWChem software package [43]. The DFT PBE96 [44] exchange correlation function was used for the majority of these calculations, however, the PBE96 + Grimme2 [44-46], and the hybrid [38] PBE0 [47] exchange correlation potentials were also used in the analysis. A recent review has proposed that Grimme corrections may be important in describing bulk water systems [46]. However, our careful comparisons of DFT results for $\mathrm{Fe}^{3+}$ and other first row transition metal aqua ions with experimental EXAFS data found that the effects of these additional corrections were quite small for solvated ions [48]. The interactions between valence electrons and the atom centers were approximated using generalized norm-conserving Hamann pseudopotentials $[49,50]$ for $\mathrm{O}$, and $\mathrm{H}$ and a norm-conserving Troullier-Martin pseudopotential [51], which contained $4 s, 4 p$, and $3 d$ projectors and a semi-core correction was used for Fe. The pseudopotentials were modified into a separable form as suggested by Kleinman and Bylander [52]. For gradient corrected calculations, the NWPW module automatically generates pseudopotentials using the specified exchange correlation functional. The original pseudopotential parameterization suggested by Hamann were slightly softened by increasing the core raii: $\mathrm{H}: \mathrm{r}_{\mathrm{cs}}=0.8$ a.u., $\mathrm{r}_{\mathrm{cp}}=0.8$ a.u.; $\mathrm{O}$ : $r_{c s}=0.7$ a.u., $r_{c p}=0.7$ a.u., and $r_{c d}=0.7$ a.u. The radial cutoffs for the Troullier-Martin pseudopotential for $\mathrm{Fe}$ were $r_{c s}=1.24$ a.u., $r_{c p}=1.24$ a.u., $r_{c d}=1.23$ a.u., and the s-channel pseudopotentials was chosen for the local potential in the Kleinman and Bylander expansion. Since this is a spin ordered system, unrestricted DFT calculations were performed. The electronic wavefunctions were expanded using a plane-wave basis with periodic boundary conditions at the $\Gamma$-point with a wavefunction cutoff energy of 100 Ry and a density cutoff energy of $200 \mathrm{Ry}$.

To establish the accuracy of the DFT PBE96 approach used in this manuscript, we evaluated its accuracy by calculating the bulk structural properties of the perfect goethite crystal. The orthorhombic unit cell contains 4 Fe atoms, 8 Oxygen atoms and 4 Hydrogen atoms (see Fig. 1). The lattice parameters were optimized using a $1 \times 3 \times 2$ supercell $\Gamma$-point calculation. Relaxing the unit cell gave lattice parameters of $10.067,9.155$, and 9.204 $\AA$ $(a=10.067 \AA, b=3.0517 \AA, c=4.6020 \AA)$, which are within $1.5 \%$ of experimental results [22, 53, 54]. These results are slightly better than found by Rosso and Rustad [55] in their DFT calculations, but not in as good agreement as those reported by Kubicki et al. [2], which were within $0.5 \%$ of experimental results [2] for lattice constants. 
Goethite is antiferromagnetic at standard temperature $(298.15 \mathrm{~K})$ and pressure (1 atm). Three different spin orderings of the magnetic $\mathrm{Fe}$ atoms were calculated ([++-], [+-+], and [+-+-] along the $a$ axis). A spin-penalty scheme was used to initialize the antiferromagnetic configurations [54]. The [+-+-] spin configuration shown in Fig. 1 was found to have the lowest energy (see Ying et al. [54]), which is in agreement with the prior calculations of Kubicki et al. [2]. These calculations predict a Fe site local spin $\mathrm{S}=3.5 / 2$ compared $\mathrm{S}=3.8 / 2$ seen in experiment [56].

To simulate the goethite (100) (or (010) Pbnm) surface a $30.0 \AA \times 9.155 \AA \times 9.204 \AA$ periodic unit cell was used for all surface calculations in this study. This cell contains a $3 \times 2(100)$ surface slab with approximately $10 \AA$ between slabs. The goethite surface slab contained $24 \mathrm{Fe}$, $48 \mathrm{O}$ and $60 \mathrm{H}$ atoms, and the thickness of the slab was $9.4 \AA$ (distance in a direction between two oxygen atoms on surface). The slab has a relatively small number of $4 \mathrm{Fe}$ layers, however, the expansion between the layers from the bulk values was observed to only be a couple of percent in these studies.

AIMD simulations were performed using open shell DFT. The system was propagated in time using CarParrinello Molecular dynamics (CPMD) scheme [40]. A plane wave basis and $\Gamma$ point sampling were used to expand DFT wavefunctions. Simulations were carried out using both the PBE96 exchange correlation potential and PBE96 plus the Grimme2 correction for dispersion to check the importance of dispersion corrections for the water-water interactions [46]. The same pseudopotential and cutoff energies used in plane-wave optimization part were used in the dynamic AIMD simulations. Equation of motions in CPMD were integrated using position Verlet algorithm, with a time step $0.12 \mathrm{fs}$ and fictitious orbital mass $600.0 \mathrm{au}$. All hydrogen atoms were replaced by deuterium to facilitate the integration. The simulation was carried out in a constant temperature canonical ensemble (300 K) using Nose-Hoover thermostats [57-59] to control the temperatures of the ions and the 1-electron orbitals.

The simulation of the solvated interface included 65 waters between the slabs (including 12 water molecules from the goethite surface). The water density in between the slabs was near $1.0 \mathrm{~g} / \mathrm{cm}^{3}$. The relatively small thickness between the slabs results in a nano-confined water layer that will not formally capture several important properties of bulk water (e.g. diffusion, dielectric relaxation response), however the water layer is expected to be large enough to describe the structure of the watersurface interface. This large simulation contained 1024 valence electrons (512 spin up electrons and 512 spin down electrons). To initiate the simulation, the 53 water molecules in between the slabs were pointed away from hydrated goethite surfaces. An initial simulation time of at 1.5 ps was performed to equilibrate the system after which trajectory snapshots were collected.

\section{Results and discussion}

Termination of (100) surface without interface water layers There are several possible surface cleavages for the goethite (100) surface in which the surface $\mathrm{Fe}^{3+}$ cations maintain the octahedral structure that is found in the bulk. Ghose et al. [22] in fitting their crystal truncation rod (CTR) data postulated four possible surface cleavages for this surface. We initially considered the two largest fractions of the surface cleavages obtained by Ghose et al. The first cleavage (surface I) shown in Fig. 2 has an $\mathrm{OH}^{-}$layer on the surface. The second cleavage (surface II) shown in Fig. 3 has an $\mathrm{O}^{2-}$ layer on the surface. These two cleavages were labeled by Kubicki et al. [2] as the $\mathrm{O}^{2-}$ termination surface and the $\mathrm{O}^{1-}$ termination surface respectively (surface $\mathrm{I} \rightarrow \mathrm{O}^{2-}$ termination, surface $\mathrm{II} \rightarrow \mathrm{O}^{1-}$ termination). The unsaturated surface $\mathrm{OH}^{-}$ and $\mathrm{O}^{2-}$ anions in both these cleavages were protonated to neutralize the charge. The first cleavage has another possible protonation (surface ID) shown in Fig. 4, which we also considered. In this cleavage, also considered by de Leeuw and Cooper [27], the third highest oxygen in the cleaved structure is protonated instead of the top hyd roxyl.

It is straightforward to compare the energies of the three surface models since they have the same number of atoms per unit cell (24 Fe atoms, $60 \mathrm{O}$ atoms and 48 $\mathrm{H}$ atoms for the $3 \times 2$ surface cells). Energetic results for the three surface models at the PBE96 level are shown in upper part of Table 1. It was found that Surface I has the lowest energy, which is in agreement with the analysis of the CTR experiments [22] and the prior DFT + U calculations [2]. The next lowest energy structure is surface II, followed by surface ID.

For the lowest energy surface, surface I, it was found that the water molecules capping each surface Fe, were very weakly bonded. Optimization of surface I resulted in a large $\mathrm{Fe}-\mathrm{OH}_{2}$ bond distance of $2.45 \AA$. This is considerably larger than $2.15 \AA$ distance in the model fitted from CTR data [22]. Kubicki et al. saw a similar result in their PBE96 + U calculations [2]. Further support of the weak interaction between water and surface $\mathrm{Fe}^{3+}$ was obtained from AIMD simulations of the isolated surface I (Fig. 2). These simulations performed at $300 \mathrm{~K}$ showed that the capping waters readily leave the surface to form a thin water layer. The other higher energy surface structures, surface II and surface ID, which were instead capped by hydroxide, had distances of 1.94 and $1.95 \AA$ respectively. These distances are significantly smaller 


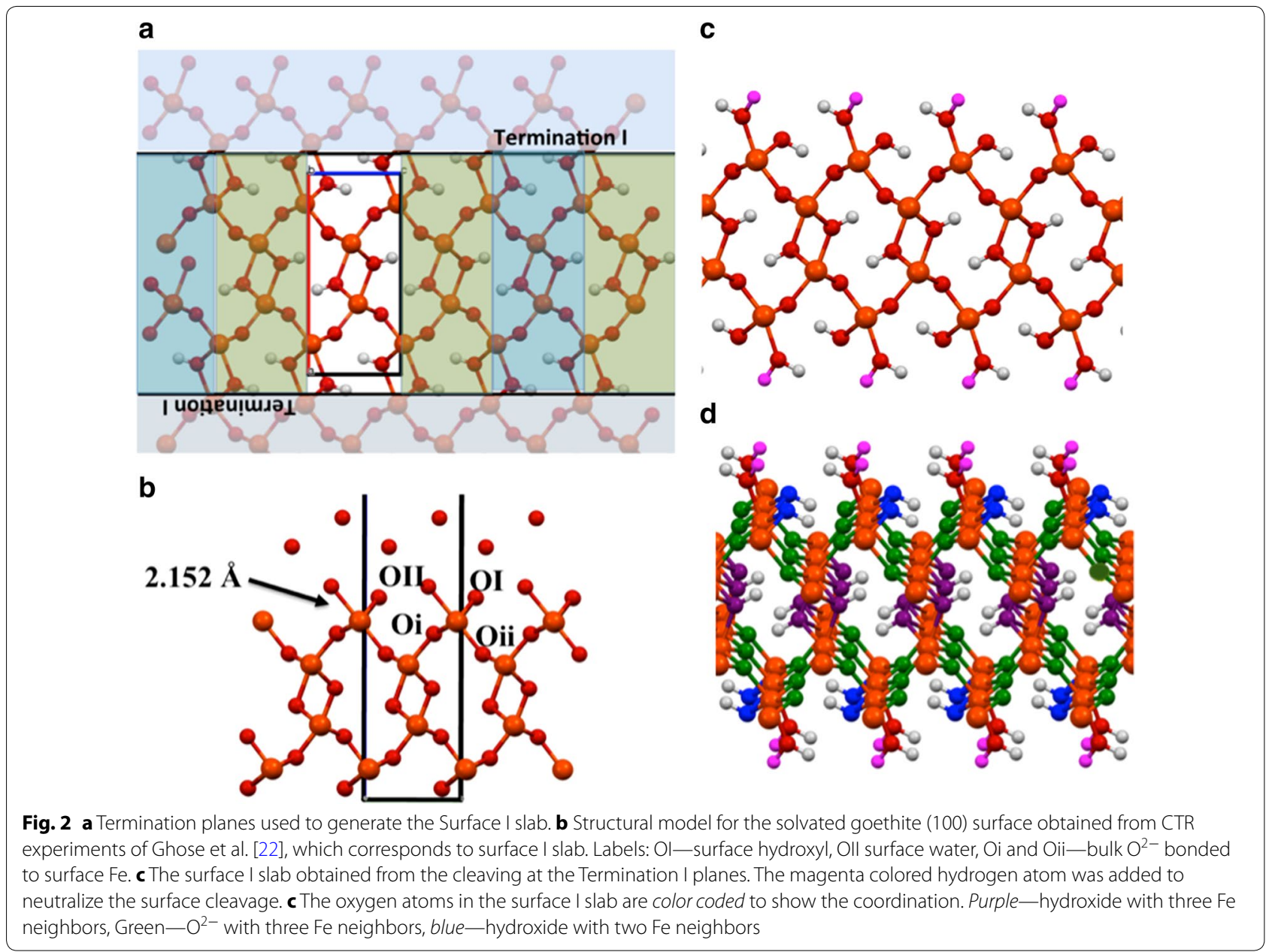

than the $2.15 \AA$ distance predicted from the CTR analysis [22] and $2.13 \AA$ distance from prior MD calculations [27].

Even though the large $\mathrm{Fe}-\mathrm{OH}_{2}$ distances for the lowest energy surface (surface I) found in DFT calculations do not agree with prior MD calculations [8, 27, 28, 33] or with the structural model fit from CTR experiments [22], the large distances make chemical sense because (in a ionic lattice model) the negative charge due to the neighboring ions near the surface $\mathrm{Fe}^{3+}$ is -3 . This makes the region near the surface $\mathrm{Fe}^{3+}$ effectively neutral, which in turn makes the Coulomb attraction to the dipole in the water molecule very small. To quantify this one can sum up the effective charge of the neighboring groups and add it to the +3 charge of the surface $\mathrm{Fe}^{3+}$ to determine its effective charging. Each surface $\mathrm{Fe}^{3+}$, without counting the surface water, is neighbors with $3 \mathrm{O}^{2-}$ that are shared by $3 \mathrm{Fe}^{3+}$ (shown as green spheres in Fig. 2c), and 2 $\mathrm{OH}^{-}$that are shared by $2 \mathrm{Fe}^{3+}$ (shown as blue spheres in Fig. 2c). This analysis shows that each surface $\mathrm{Fe}^{3+}$ is surrounded by a valence charge of $3(-2) / 3+2(-1) / 2=-3$ with only 5 neighbors. On the other hand, the bulk $\mathrm{Fe}^{3+}$ atoms are neighbors with $3 \mathrm{OH}^{-}$shared by $3 \mathrm{Fe}^{3+}$ and $3 \mathrm{O}^{2-}$ shared by $3 \mathrm{Fe}^{3+}$, resulting in a valence charge of $3(-1) / 3+3(-2) / 3=-3$. This simple analysis clearly shows that the long surface $\mathrm{Fe}-\mathrm{OH}_{2}$ distance can be rationalized by the fact that the surface $\mathrm{Fe}^{3+}$ atoms are already fully charge balanced (i.e. valence saturated) with only 5 neighbors.

A theoretical approach that formalizes the charge balance arguments above is bond valence theory [39, 6062]. This theory, originally developed by Pauling [63], is widely used to predict bond lengths in minerals. The basic supposition of this theory is that the bond strengths around a cation (or anion) are set equal to the valence of the cation (anion) divided by its coordination number (assuming the same ligands). For example, each bond about an octahedral $\mathrm{Fe}^{3+}$ has a bond strength of $3 / 6$ or $1 / 2$. Similarly the bond strengths of the $\mathrm{O}-\mathrm{H}$ bonds in a water molecule would be $2 / 2$ or 1 , since the oxygen atom has an oxidation state of -2 or in terms of valence is 2 . The theory can be extended to predict bond lengths by further 

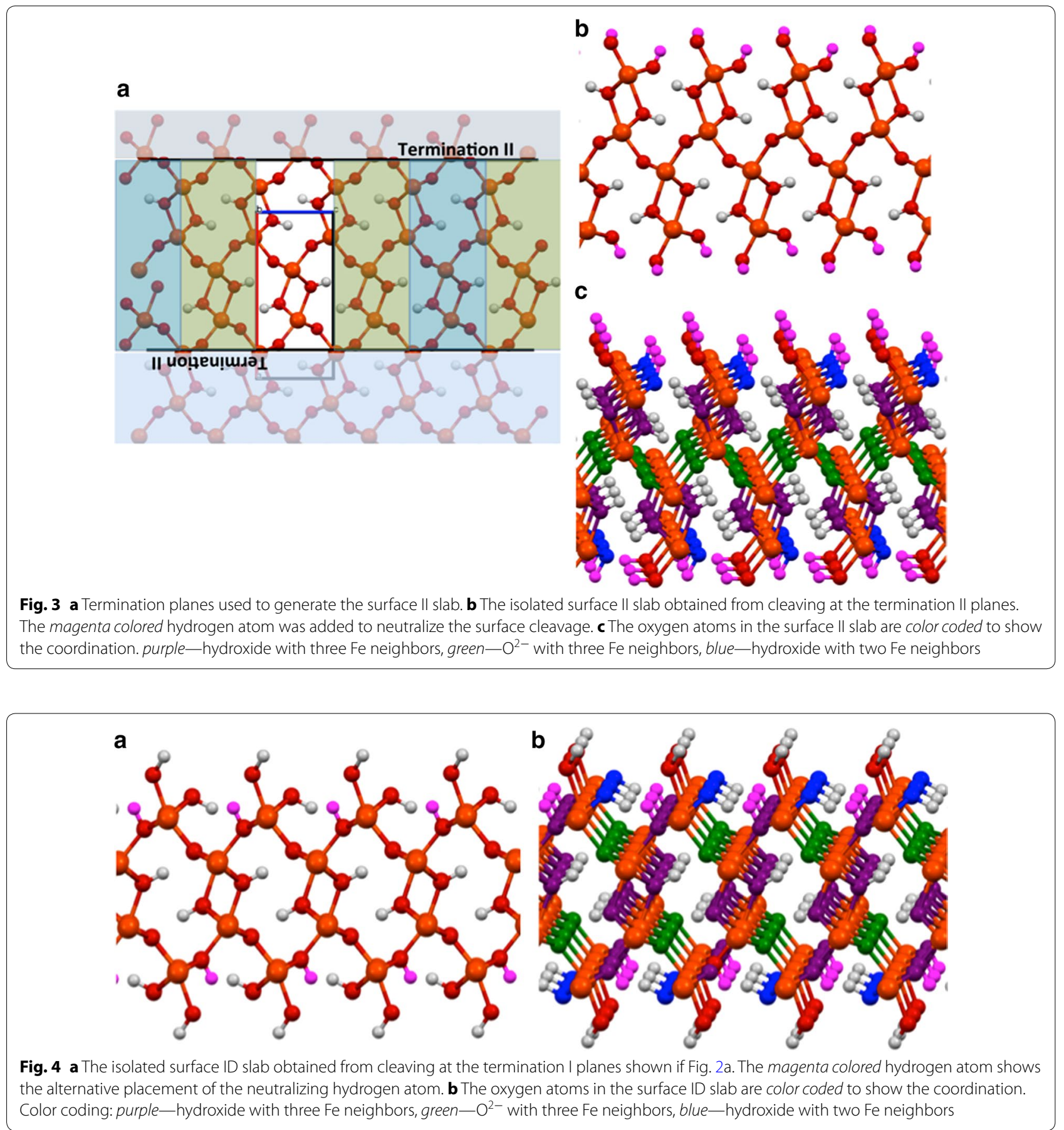

assuming that the bond strengths are inversely related to the bond lengths with the following formula

$$
v_{i j}=\exp \left(\frac{R_{i j}^{0}-R_{i j}}{B}\right)
$$

where $v_{i j}$ is the bond strength and $R_{i j}$ is the bond length between atoms $i$ and $j, R_{i j}^{0}$ is the distance for a bond between atom $i$ and $j$ to have a bond strength equal to 1 , and $B$ is an empirical constant. Brown and Altermatt $[39,60]$ have determined $R_{i j}^{0}$ for each kind cation-anion in the Inorganic Crystal Structure Data Base (ICSD) [64] in which the anions are chemically identical. The fitted values of $R_{i j}^{0}$ for $\mathrm{Fe}^{3+}-\mathrm{O}^{2-}$ and $\mathrm{H}^{+}-\mathrm{O}^{2-}$ are 1.759 and $0.882 \AA$ respectively. During their fitting they also found that $B$ varies very little from structure to structure, and 
Table 1 Total and relative energies from DFT PBE96 simulations for the vacuum surface models and solvated surfaces models of the (100) surface of goethite

\begin{tabular}{|c|c|c|c|}
\hline Surface model & Molecular formula of supercell & Average total energy (a.u.) & Relative energy ( $\mathrm{kJ} / \mathrm{mol})$ \\
\hline \multicolumn{4}{|l|}{ Vacuum surface models } \\
\hline $3 \times 2$ surface 1 & $\mathrm{Fe}_{24} \mathrm{O}_{60} \mathrm{H}_{48}$ & -1660.543130 & 0 \\
\hline $3 \times 2$ surface $\|$ & $\mathrm{Fe}_{24} \mathrm{O}_{60} \mathrm{H}_{48}$ & -1660.379184 & 430.6 \\
\hline $3 \times 2$ surface ID & $\mathrm{Fe}_{24} \mathrm{O}_{60} \mathrm{H}_{48}$ & -1660.328303 & 564.3 \\
\hline \multicolumn{4}{|l|}{ Solvated surface models } \\
\hline $3 \times 2$ surface $\mathrm{I}+53 \mathrm{H}_{2} \mathrm{O}$ & $\mathrm{Fe}_{24} \mathrm{O}_{113} \mathrm{H}_{154}$ & -2570.717514 & 0 \\
\hline $3 \times 2$ surface $\mathrm{II}+53 \mathrm{H}_{2} \mathrm{O}$ & $\mathrm{Fe}_{24} \mathrm{O}_{113} \mathrm{H}_{154}$ & -2570.566486 & 397.7 \\
\hline $3 \times 2$ surface ID $+53 \mathrm{H}_{2} \mathrm{O}$ & $\mathrm{Fe}_{24} \mathrm{O}_{113} \mathrm{H}_{154}$ & -2570.498789 & 574.5 \\
\hline
\end{tabular}

they suggested using a fixed value of $B=0.37 \AA$. Setting the valences of each atom, $V_{i}$, equal to the sum of its bond strengths

$$
V_{i}=\sum_{j} v_{i j} \quad \text { or } \quad V_{i}=\sum_{j} \exp \left(\frac{R_{i j}^{0}-R_{i j}}{B}\right)
$$

generates a system of non-linear equations for the bonding network, which can be solved for all the bond distances in the structure In goethite, the valences for $\mathrm{Fe}^{3+}$, $\mathrm{O}^{2-}$ and $\mathrm{H}^{+}$are 3, 2 and 1 respectively. There are several strategies for solving this system of non-linear equations for the bond distances. We chose to solve them by minimizing the following penalty function

$$
\chi=\sum_{i}\left(V_{i}-\sum_{\substack{j \\ \text { əi,jform bonds }}} \exp \left(\frac{R_{i j}^{0}-R_{i j}}{B}\right)\right)^{2}
$$

with respect to $R_{i j}$. The results of these calculations for the $3 \times 2$ Surface I model are given in the second column of Table 2 and show that the surface $\mathrm{Fe}-\mathrm{OH}_{2}$ distances are very long and essentially unbound.
It is also possible that electronic structure effects missing from the PBE96 exchange correlation functional may be able to contract the large $\mathrm{Fe}-\mathrm{OH}_{2}$ distances. It is well know that DFT has major problems predicting the electronic structure near the Fermi level (e.g. band gap) [54] for transition metal oxides and oxyhydroxides and other strongly correlated systems. These errors at the Fermi level can sometimes, but not always, produce anomalous structures, e.g. including exact exchange might effect the charge distribution between the metal and oxygen atoms and, therefore the bond formation of the water to the surface. To check for these possible effects, hybrid DFT PBE0 (presumed to be a better level of exchange correlation in DFT [38]) were carried out for the $3 \times 2$ Surface I model. In addition, DFT as mean field theory cannot correctly treat long-range dispersion forces and as a result dispersion forces can be underestimated. To estimate the effects of dispersion, PBE96 + Grimme2 [46] calculations were also carried out for the $3 \times 2$ Surface I model. These calculations, results given in Table 2, also lead to large $\mathrm{Fe}-\mathrm{OH}_{2}$ distances $(2.45 \AA$ PBE96, $2.39 \AA$ PBE96 + Grimme2, and $2.41 \AA$ PBE0). This suggests that the prediction of large distances is not overly sensitive to the level of theory used in the electronic structure calculations. These calculations clearly show that there is a

\begin{tabular}{|c|c|c|c|c|c|}
\hline Surface $\mathrm{Fe}-\mathrm{O}$ distances & BV & PBE96 & PBE96 + Grimme2 & PBEO & Exp. CTR model \\
\hline $\mathrm{Fe}-\mathrm{OH}_{2}(\mathrm{Fe}-\mathrm{OII})$ & Unbound & 2.45 & 2.39 & 2.41 & 2.15 \\
\hline $\mathrm{Fe}-\mathrm{OH}(\mathrm{Fe}-\mathrm{Ol})$ & 2.02 & 2.10 & 2.11 & 2.09 & 2.09 \\
\hline Fe-O (Fe-Oi, Fe-Oii) & $1.91,1.91$ & $2.01,1.95$ & $2.01,1.94$ & $2.01,1.98$ & $1.95,1.97$ \\
\hline 1st layer Fe-O distances & BV & PBE96 & PBE96 + Grimme2 & PBE0 & CTR model \\
\hline $\mathrm{Fe}-\mathrm{OH}$ & $2.17,2.17,2.17$ & $2.14,2.14,2.19$ & $2.15,2.15,2.16$ & $2.13,2.13,2.16$ & $2.19,2.19,2.10$ \\
\hline $\mathrm{Fe}-\mathrm{O}$ & $1.91,1.91,1.91$ & $1.94,2.01,2.01$ & $1.93,2.00,2.00$ & $1.98,2.00,2.00$ & $1.94,1.93,1.93$ \\
\hline
\end{tabular}

Table 2 Fe-O bond distances for the surface I slab model of the (100) surface of goethite

See Fig. 4c for definitions of OI, OII, Oi, and Oii oxygens 
strong disagreement in the results for the $\mathrm{Fe}-\mathrm{OH}_{2}$ distances using different theories. Electronic structure calculations and bond valence theory calculations predict large distances, and prior classical molecular dynamics potentials and the model fitted to CTR experiments [22] predicts distances that are just slightly larger $(\sim 2.15 \mathrm{~A} \AA)$ than the bulk distances for $\mathrm{Fe}-\mathrm{OH}$ in goethite $(\sim 2.09 \AA)$.

\section{AIMD simulations of goethite (100) water interface}

To further check the relative energetics of the three terminations and resulting surfaces we added $53 \mathrm{H}_{2} \mathrm{O}$ molecules between the slabs and ran AIMD simulations using the PBE96 exchange correlation functional for at least 10 ps. Each of three simulations was performed using a unit cell that contained a total of $24 \mathrm{Fe}$ atoms, $113 \mathrm{O}$ atoms and $154 \mathrm{H}$ atoms $\left(\mathrm{Fe}_{24} \mathrm{O}_{60} \mathrm{H}_{48}+53 \mathrm{H}_{2} \mathrm{O}\right)$. The simulations were designed to have water densities in between the slabs near $1.0 \mathrm{~g} / \mathrm{cm}^{3}$. The average potential energies, given in the lower part of Table 1 , show that the solvated surfaces have nearly the same relative energetics as the vacuum terminated surfaces. Given that Surface I was found to have a significantly lower energy than the other two surface models in vacuo and with solvation, the rest of the manuscript only presents results for this surface.

\section{The structure of the surface I + water interface}

Simulations were carried out for this surface using both the PBE96 and PBE96 + Grimme2 exchange correlation functionals. The laterally averaged densities per supercell of the Fe and O atoms for the PBE96 and PBE96 + Grimme2 AIMD simulations are given in Fig. 5. Both the simulations produce well-defined and isolated peaks for the oxygen and iron densities for the goethite slabs, which are in the ranges between -20 to $-9 \AA$, and 9 to $20 \AA$. In the optimized crystal at the PBE96 level the planes of iron atoms were separated by $2.08,2.96$, and $2.08 \AA$. Whereas in the slab, the distances between
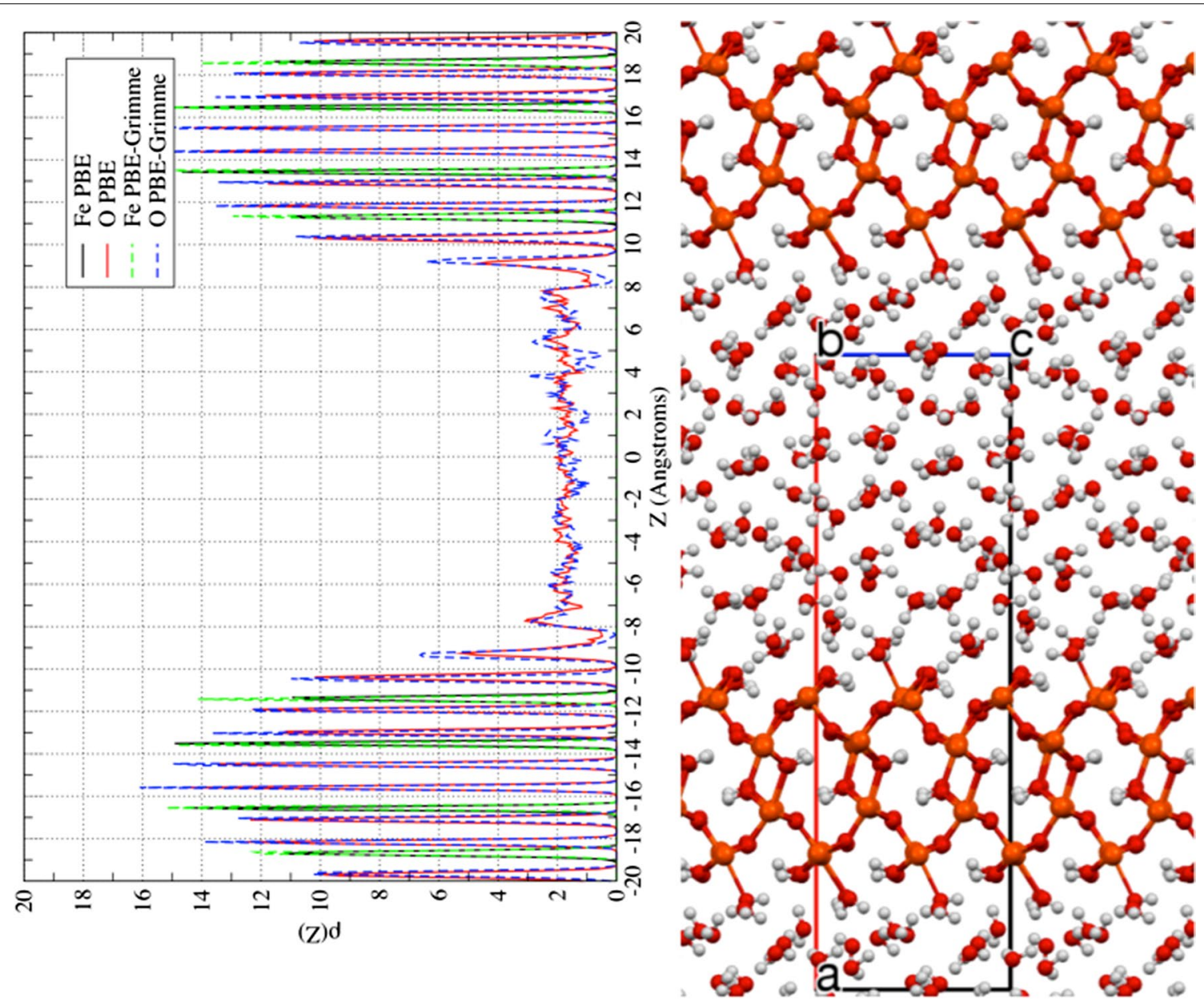

Fig. 5 Left Laterally averaged oxygen atom and iron atom densities from PBE96 and PBE96 + Grimme2 AIMD simulations. Right Snapshot of PBE96 AIMD simulation aligned with densities 
the planes were slightly expanded to be $2.11,3.07$, and $2.11 \AA$ at the PBE96 level, and 2.13, 2.98, and $2.13 \AA$ at the PBE96 + Grimme2 level. This results in an overall expansion of 2.3 and $1.6 \%$ between the outer planes of iron atoms at the PBE96 and PBE96 + Grimme2 levels respectively. This is in reasonable agreement given the relatively small slab models used in this study. Similarly the distances between the planes of oxygen atoms in the slab were slightly larger than found for the bulk. The oxygen planes were separated by 1.44, 1.07, 1.44, 1.08, 1.44, 1.07, 1.44 $\AA$, for the bulk PBE96 crystal; 1.54, 1.07, 1.52, $1.08,1.52,1.07$, and 1.54 Å for the slab at the PBE96 level; and $1.48,1.09,1.47,1.09,1.48,1.09$ and $1.48 \AA$ for the slab at the PBE96 + Grimme2 level.

The first outside layer of oxygen atoms (OI) located at $\mathrm{z} \approx-10.4 \AA, 10.4 \AA$ were the hydroxides bonded to the surface Fe atoms (see Fig. 4c for definition of oxygen labels). The integration of these peaks produced exactly 6 oxygen atoms for the PBE96 and PBE96 + Grimme2 simulations. The second outside layer of oxygen atoms (OII) located at $\mathrm{z} \approx-9.0 \AA, 9.0 \AA$ were the water molecules attached to the surface Fe atoms. This layer of water molecules was loosely bound to the surface, and the bond between the water $\mathrm{O}$ and the surface $\mathrm{Fe}^{3+}$ were observed to frequently break and reform during the simulations. In some cases these water molecules exchange with the more bulk-like water molecules of the next layer. The integration of these peaks produced 4.63 and 5.98 oxygen atoms for the PBE96 and PBE96 + Grimme2 simulations respectively. The first inside layer of oxygen atoms (Oi) located at $\mathrm{z} \approx-11.9 \AA, 11.9 \AA$ were $\sim 180^{\circ}$ away from the OI oxygen atoms, and the second inside layer of oxygen atoms (Oii) located at $\mathrm{z} \approx-13.0 \AA$, $13.0 \AA$ 就e nearly $\sim 180^{\circ}$ away from the OII oxygen atoms.

In Table 3, the surface $\mathrm{Fe}-\mathrm{O}$ bond distances and $\mathrm{O}-$ $\mathrm{Fe}-\mathrm{O}$ bond angles for the goethite (100) surface obtained from PBE96 AIMD and PBE96 + Grimme2 AIMD along with values extracted from published experimental data are given. The maximum in the first shell peaks of $\mathrm{Fe}-\mathrm{O}$ histogram shown in Fig. 6 were used for the FeOII distances. Except for the Fe-OII distance, the bond distances and angles for the surface slab were in in good agreement with the fitted model from CTR experiments [22]. While the Fe-OII bond distances did contract somewhat from the vacuum surface, the distances of 2.40 and $2.34 \AA$ from the PBE96 and PBE96 + Grimme2 theories respectively, were still considerably larger than $2.15 \AA$ in the model fitted from CTR data and the $2.21 \AA$ micro-solvated MD calculations. The average percent difference for the rest of bond lengths with respect to the CTR values was 1.7 and $1.1 \%$ for the PBE96 and PBE96 + Grimme2 theories respectively, and for the bond angles was 2.7 and $3.1 \%$.

\section{Electronic structure of interfacial water}

The proximity of the surface waters to the strongly ionic structure of the goethite surface can change the properties of solvating waters. These complex electronic structure effects are automatically included in AIMD simulations via the use of the Schrödinger equation to calculate the interatomic forces and leads to changes in the electronic structure of the surface waters. To characterize these effects we analyzed the difference in density of the full $\mathrm{Fe}_{24} \mathrm{O}_{60} \mathrm{H}_{48}+53 \mathrm{H}_{2} \mathrm{O}$ PBE96 AIMD system (or $\mathrm{Fe}_{24} \mathrm{O}_{48} \mathrm{H}_{24}+65 \mathrm{H}_{2} \mathrm{O}$ if OII water not part of slab) minus the $\mathrm{Fe}_{24} \mathrm{O}_{48} \mathrm{H}_{24}$ slab and the water layers (including the OII water) is shown in Fig. 7. The polarization of the water molecules in the interface layer due to the surface (and vice versa) is fairly small and localized only in the immediate vicinity of the interface, and it is concentrated in between the atoms near the OI hydroxyls and the OII waters. Note that changes in density (polarization) are also present in the solid surface region.

To provide a more quantifiable measure of the polarization we performed a detailed analysis of the electronic structure of the water molecules in terms of the positions of the centers of their Boys localized bond orbitals Table 3 Comparison of surface structure parameters
for the (100) surface goethite + water between PBE96
AIMD and PBE96 + Grimme2 AIMD simulations
of $\mathrm{Fe}_{24} \mathrm{O}_{60} \mathrm{H}_{48}+53 \mathrm{H}_{2} \mathrm{O}$ and fitted structure from CTR
experiments of Ghose et al. [22]

\begin{tabular}{|c|c|c|c|}
\hline $\begin{array}{l}\text { Surface structure } \\
\text { parameters }\end{array}$ & PBE96 AIMD & $\begin{array}{l}\text { PBE96 + Grimme2 } \\
\text { AIMD }\end{array}$ & $\begin{array}{l}\text { Exp. CTR } \\
\text { model }\end{array}$ \\
\hline $\mathrm{R}(\mathrm{Fe}-\mathrm{OII})$ & $\sim 2.40$ & $\sim 2.34$ & 2.152 \\
\hline \multicolumn{4}{|l|}{$\sigma(\mathrm{Fe}-\mathrm{OII})$} \\
\hline $\mathrm{R}(\mathrm{Fe}-\mathrm{OI})$ & 2.10 & 2.11 & 2.097 \\
\hline$\sigma(\mathrm{Fe}-\mathrm{Ol})$ & 0.08 & 0.08 & \\
\hline $\mathrm{R}(\mathrm{Fe}-\mathrm{Oi})$ & 2.02 & 2.00 & 1.950 \\
\hline$\sigma(\mathrm{Fe}-\mathrm{Oi})$ & 0.07 & 0.08 & \\
\hline R(Fe-Oii) & 1.94 & 1.97 & 1.966 \\
\hline$\sigma(\mathrm{Fe}-\mathrm{Oii})$ & 0.06 & 0.07 & \\
\hline$\angle \mathrm{OI}-\mathrm{Fe}-\mathrm{OII}$ & 80.2 & 81.1 & 81.25 \\
\hline$\sigma \angle \mathrm{Ol}-\mathrm{Fe}-\mathrm{OII}$ & 5.5 & 5.3 & \\
\hline$\angle \mathrm{OII}-\mathrm{Fe}-\mathrm{Oi}$ & 87.7 & 87.7 & 82.85 \\
\hline$\sigma \angle \mathrm{Oll}-\mathrm{Fe}-\mathrm{Oi}$ & 5.8 & 5.7 & \\
\hline$\angle \mathrm{Oll}-\mathrm{Fe}-\mathrm{Oii}$ & 170.4 & 170.3 & 178.89 \\
\hline$\sigma \angle \mathrm{Oll}-\mathrm{Fe}-\mathrm{Oii}$ & 4.9 & 5.0 & \\
\hline$\angle \mathrm{Ol}-\mathrm{Fe}-\mathrm{Oi}$ & 81.8 & 82.6 & 80.85 \\
\hline$\sigma \angle \mathrm{OI}-\mathrm{Fe}-\mathrm{Oi}$ & 3.6 & 3.4 & \\
\hline$\angle \mathrm{OI}-\mathrm{Fe}-\mathrm{Oii}$ & 97.1 & 94.5 & 99.5 \\
\hline$\sigma \angle \mathrm{Ol}-\mathrm{Fe}-\mathrm{Oii}$ & 5.7 & 4.7 & \\
\hline$\angle \mathrm{OIII-Fe-Oii}$ & 97.6 & 97.1 & 96.5 \\
\hline$\sigma \angle \mathrm{OIII}-\mathrm{Fe}-\mathrm{Oii}$ & 4.8 & 4.3 & \\
\hline
\end{tabular}

See Fig. 4c for definitions of OI, OIl, Oi, and Oii oxygens (length unit: $\AA$, angle unit: ${ }^{\circ}$ ) 


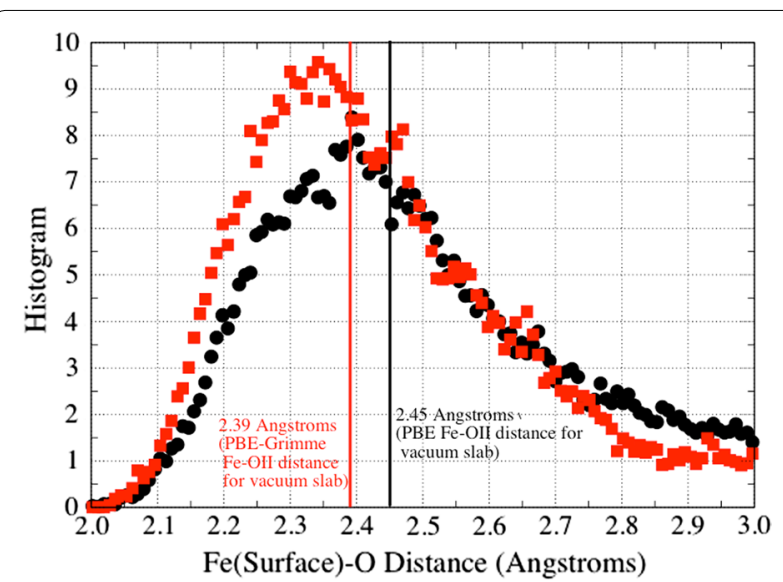

Fig. 6 Histograms of surface Fe-Oll distance from PBE96 and PBE96 + Grimme2 AIMD simulations. See Figs. 4C and 7 for definition of Oll

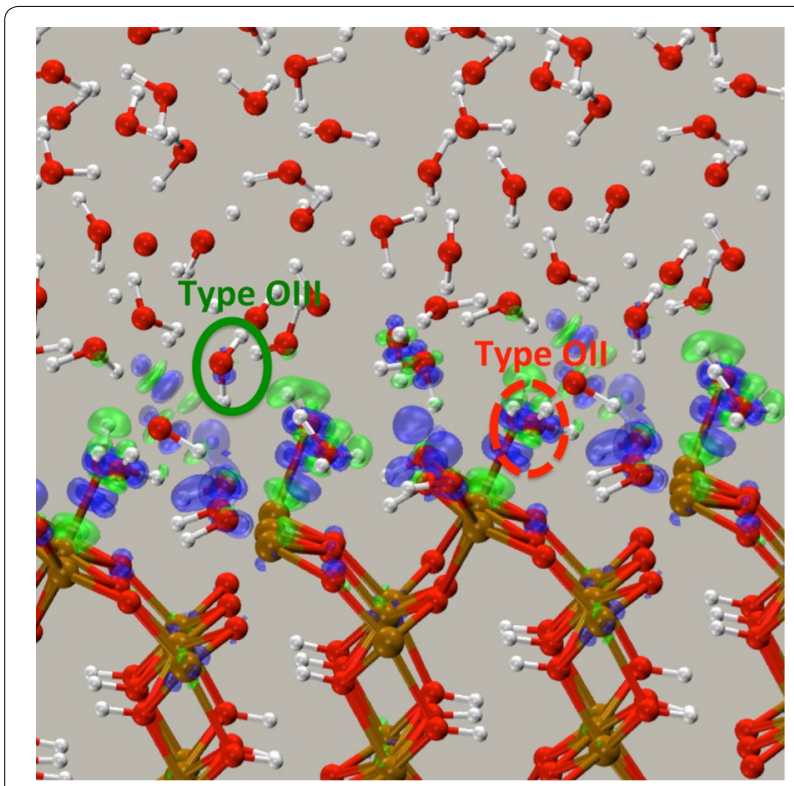

Fig. 7 Isosurfaces of the difference in density of the full $\mathrm{Fe}_{24} \mathrm{O}_{48} \mathrm{H}_{24}+65 \mathrm{H}_{2} \mathrm{O}$ PBE96 AIMD system minus the $\mathrm{Fe}_{24} \mathrm{O}_{48} \mathrm{H}_{24}$ slab and the water layers (including the Oll water) is shown. The surplus charge distribution is shown in the transparent blue: 0.005 a.u. isosurface, and the deficit charge distribution is shown in the transparent green: -0.005 a.u. isosurface. Configuration taken from the end of a 10 ps AIMD simulation

relative to the oxygen center of the water molecule (Wannier-Boys function center, WBFC, i.e. center of the Boys orbital). This representation has been shown by Lightstone et al. [65] and Bogatko et al. [66] to provide a measure of metal ion-water interactions. If there is a significant impact of the presence of the surface on the electronic structure of a water molecule, the distance from the $\mathrm{O}$ center to the WBFC of the lone pair orbitals
(LPO) and the bond orbitals (BO) between the $\mathrm{O}$ and $\mathrm{H}$ atoms, $\mathrm{d}_{\mathrm{O} \text {-wfc }}$ and the angle formed by the WBFCs and the $\mathrm{O}$ center $\Theta_{\text {wfc-O-wfc }}$ (see Fig. 8 for definitions) in the molecule should be altered relative to those found in bulk water. The results of this analysis, in which $\mathrm{d}_{\mathrm{O}-\mathrm{wfc}}$ and $\Theta_{\text {wfc-O-wfc }}$ are taken as averages of several independent snapshots from the $\mathrm{Fe}_{24} \mathrm{O}_{60} \mathrm{H}_{48}+53 \mathrm{H}_{2} \mathrm{O}$ PBE96 AIMD simulation, are shown in Table 4. For comparison the WBFC analysis for the homogeneous $\mathrm{Fe}^{3+}-64$ water, $\mathrm{Al}^{3+}-64$ water and the 64 water simulations from Bogatko et al. [66] are also given in the table. The analysis shows that both the spin up and spin down electrons in oxygen from type OIII water have values for $\mathrm{d}_{\mathrm{O}-\mathrm{wf}}$ and $\Theta_{\text {wfc-O-wfc }}$ similar to those of bulk water molecules. The values for type OII water are also very close to bulk water values. However, there are small but noticeable differences. For this water the $\mathrm{d}_{\mathrm{O} \text {-wf }}$ for spin down LPO has been lengthened by $\sim 0.02 \AA$, whereas the $\mathrm{d}_{\mathrm{O} \text {-wfc }}$ for spin up LPO is essentially the same as bulk water. This is consistent with the weak bonding of these water molecules to the surface. Plots of the spin up and spin down LPOs (not shown) also show that the spin down LPO moves closer to the surface Fe atom whereas the spin up LPO does not. This spin dependent polarization has previously been demonstrated by Bogatko et al. [66] in the $\mathrm{Fe}^{3+}-64$ water system and is caused by the spin localization of the $\mathrm{d}$ electrons in $\mathrm{Fe}$ site because the spin down d orbitals for $\mathrm{Fe}^{3+}$ ion are empty. The effect is significantly stronger in the aqueous phase because the $\mathrm{Fe}^{3+}$ charge is not neutralized as it is on the surface. In the aqueous phase case the $d_{O-w f c}$ for spin up LPO is essentially the same as for the bulk water (the spin up d orbitals for the $\mathrm{Fe}^{3+}$ ion are completely filled). A decrease of $8^{\circ}$ and $3.4^{\circ}$ has been observed for $\Theta_{\text {wfc-O-wfc }}$ spin down and spin up LPO, respectively. The $\mathrm{BOs}$ for the OII water have also been influenced by the polarization of LPO; the $\Theta_{\text {wf-O-wfc }}$ for $\mathrm{BO}$ slightly increased and the $\mathrm{d}_{\mathrm{O}-\mathrm{wfc}}$ slightly decreased.

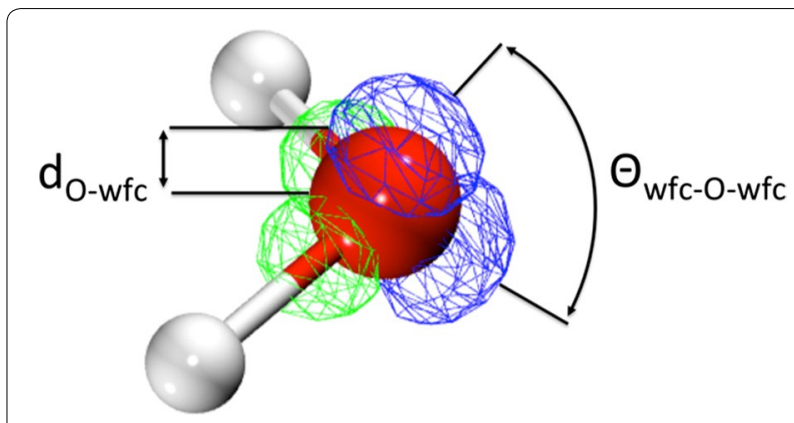

Fig. 8 Illustration of the distance from the O center to a LPO WFC, $\mathrm{d}_{\mathrm{O}-\mathrm{wfC}}$ and the angle formed by the LPO WFCs and the O center $\Theta_{\text {wfc- }}$ o-wfc Green Wannier orbitals are bonding orbitals $(\mathrm{BO})$ and the blue Wannier orbitals are the lone pair orbitals (LPO) 
Table 4 Electronic structure for the Solvation shells represented by spin up (a), Spin down ( $\beta$ ) Wannier Function Centers (WFC)

\begin{tabular}{|c|c|c|c|c|c|c|c|c|c|c|c|c|}
\hline & \multicolumn{4}{|c|}{ Oll water } & \multicolumn{4}{|c|}{ Olll water } & \multicolumn{4}{|c|}{ Bulk water } \\
\hline & \multicolumn{2}{|c|}{ dO-wfe } & \multicolumn{2}{|c|}{ Owfc-O-wfc } & \multicolumn{2}{|c|}{ dO-wfc } & \multicolumn{2}{|c|}{ Owfc-O-wfc } & \multicolumn{2}{|c|}{ dO-wfc } & \multicolumn{2}{|c|}{ Owfc-O-wfc } \\
\hline & BO & LPO & BO & LPO & BO & LPO & BO & LPO & BO & LPO & BO & LPO \\
\hline Interface water a & 0.49 & 0.33 & 104 & 116 & 0.50 & 0.33 & 103 & 119 & 0.50 & 0.33 & 103 & 118 \\
\hline \multirow[t]{4}{*}{ Interface water $\beta$} & 0.49 & 0.35 & 105 & 111 & 0.50 & 0.33 & 103 & 119 & 0.50 & 0.33 & 103 & 118 \\
\hline & \multicolumn{4}{|c|}{ First hydration shell } & \multicolumn{4}{|c|}{ Second hydration shell } & \multicolumn{4}{|l|}{ Bulk } \\
\hline & \multicolumn{2}{|c|}{ dO-wfc } & \multicolumn{2}{|c|}{ Owfc-O-wfc } & \multicolumn{2}{|c|}{ dO-wfc } & \multicolumn{2}{|c|}{ Owfc-O-wfc } & \multicolumn{2}{|c|}{ dO-wfc } & \multicolumn{2}{|c|}{ Owfc-O-wfc } \\
\hline & BO & LPO & BO & LPO & BO & LPO & BO & LPO & BO & LPO & BO & LPO \\
\hline $\mathrm{Fe}^{3+}-64 \mathrm{H}_{2} \mathrm{Oa}$ & 0.48 & 0.32 & 106 & 112 & 0.50 & 0.33 & 104 & 118 & 0.50 & 0.33 & 103 & 119 \\
\hline $\mathrm{Fe}^{3+}-64 \mathrm{H}_{2} \mathrm{O} \beta$ & 0.48 & 0.38 & 110 & 92 & 0.50 & 0.33 & 104 & 118 & 0.50 & 0.33 & 103 & 119 \\
\hline $\mathrm{Al}^{3+}-64 \mathrm{H}_{2} \mathrm{O}$ & 0.48 & 0.34 & 106 & 110 & 0.50 & 0.33 & 103 & 117 & 0.50 & 0.32 & 103 & 119 \\
\hline $64 \mathrm{H}_{2} \mathrm{O}$ & & & & & & & & & 0.50 & 0.33 & 106 & 115 \\
\hline
\end{tabular}

See Fig. 7 for definitions of Oll water and OIII water (length unit: $\AA$, angle unit: ${ }^{\circ}$ )

These results suggest that the electronic structure of the water molecules beyond the OII water molecules has returned to that of the bulk. Even the OII interfacial waters, which are often considered part of the surface, showed very little polarization presumably because the surface has a neutral charge.

\section{Dynamic processes on the interface}

No proton transfer was observed in our AIMD simulations. While fast rates of proton exchange have been observed for some water-metal oxides interfaces [67], in particular for surfaces that are highly polarizing. Whereas for the goethite (100) surface, the lack of water dissociation at the surface is consistent with the weak interaction of the interfacial OII water to the surface, as shown in the previous section. However, by having the OII water being a weakly bound means that the barrier to it exchanging with another bulk water is likely to be low, and not surprisingly several water exchanges were found to take place between bulk water molecules and the OII water molecules (i.e. the $\mathrm{Fe}-\mathrm{OH}_{2}$ ) on the surface in our $10 \mathrm{ps}$ of simulation. We estimated the exchange rate for one $\mathrm{Fe}-\mathrm{OH}_{2}$ bond on surface in the PBE96 AIMD simulation to be 0.025 exchanges/ps. However, there were not enough exchanges to calculate an accurate rate value. The ligand exchange mechanism has being widely studied for aqua ions in solutions systems [68-73]. Langford and Gray [71] proposed that substitution events may be classified into three catagories depending on the dynamic nature initiating the reaction, associative (A) (reaction initiated by one water leaving the 1st hydration shell), dissociative (D) (reaction initiated by water entering the 1st shell) and interchange (I) (simultaneous exchange of water in first hydration shell). Using this classification the exchanges we observed may be classified as dissociative (D). The process can be described as a two-step process in which the $\mathrm{Fe}-\mathrm{OH}_{2}$ bond on the surface breaks first, followed by a bulk water molecule approaching the surface $\mathrm{Fe}$ atom. An example of a D exchange process is shown in Fig. 9. This figure shows that starting from $4 \mathrm{ps}$, the $\mathrm{Fe}-\mathrm{OH}_{2}$ bond becomes unstable, and then from 5.5 to $6.5 \mathrm{ps}$, a second water molecule approaches the $\mathrm{Fe}$ atom while the initial water molecule slowly escapes. After $6.5 \mathrm{ps}$ (or $2.5 \mathrm{ps}$ in duration) the exchange process is complete.

\section{Conclusion}

DFT optimization (including PBE96, PBE96 + Grimme2, PBE0) and AIMD simulations (PBE96 and PBE96 + Grimme2) were carried out for the anhydrous goethite (100) surface and the goethite $(100)+$ water

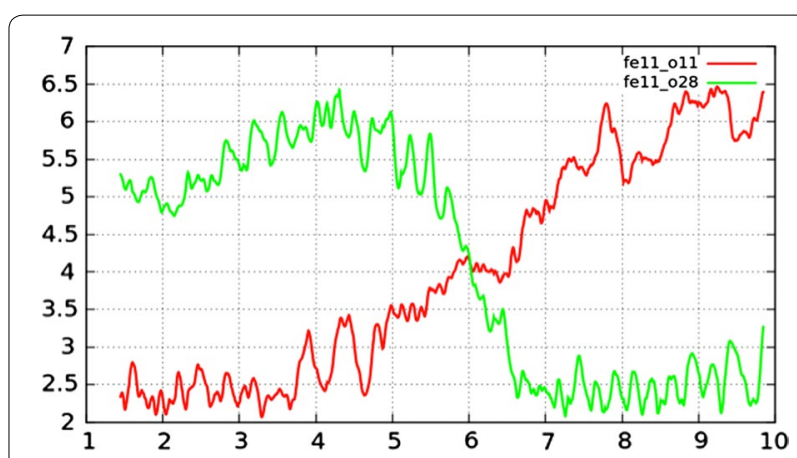

Fig. 9 Exchange of Fe bonded Oll water molecule on goethite (100) surface with bulk water 
interface. The simulations were performed for a $(3 \times 2)$ surface slab containing 65 water molecules between the slabs (near $1 \mathrm{~g} / \mathrm{cm}^{3}$ ) at temperature of $300 \mathrm{~K}$.

The surface energies calculated from DFT PBE96 optimization of three likely fully solvated neutral surface terminations in which the $\mathrm{Fe}^{3+}$ cations remain in an octahedral coordination, were compared. It was found that the lowest energy surface containing an exposed surface $\mathrm{Fe}^{3+}$ is capped by a (weakly bound) water molecule and shares a hydroxide with a neighboring $\mathrm{Fe}^{3+}$ (see surface I in Fig. 2). This surface termination agrees with the assumed largest fraction surface found in fitting CTR data by Ghose et al. [22]. The other two surfaces examined, which were capped by two hydroxides, were found to be approximately 27 and $34 \mathrm{~kJ} / \mathrm{mol}$ less stable per $1 \times 1$ surface slab (or 431 and $564 \mathrm{~kJ} / \mathrm{mol}$ for the $3 \times 2$ surface slab). The solvation of the slabs with $53 \mathrm{H}_{2} \mathrm{O}$ molecules had little effect on the relative surface energetics between the slabs. The solvated $3 \times 2$ slabs capped by two hydroxides (surface II and surface IID in Figs. 3 and 4) were found to have average energies that were 398 and $575 \mathrm{~kJ} / \mathrm{mol}$ higher then the solvated Surface I slab $(3 \times 2$ surface slab, Fig. 2).

For the most stable surface it was found that the water molecule formed by protonation of an unsaturated $\mathrm{OH}$ from the termination, and capping a surface Fe was very loosely bound (see Fig. 2). The resulting optimized or equilibrated long $\mathrm{Fe}^{3+}-\mathrm{OH}_{2}$ bond length does not agree with prior results obtained from fitting CTR data or from prior MD simulations [8, 27, 28, 33], which predicted $\mathrm{Fe}-\mathrm{OH}_{2}$ distances only slightly longer than the bulk goethite $\mathrm{Fe}-\mathrm{OH}$ distances. However, this result does agree with the prior DFT $+\mathrm{U}$ optimizations of Kubicki et al. [2]. Furthermore, the DFT PBE96 results were checked using hybrid PBE0 and dispersion corrected PBE96 + Grimme2 exchange correlation functionals and the results were found to change very little (weak bonding prediction upheld). These results support a terminated surface model in which the $\mathrm{Fe}^{3+}$ ion in the surface is coordinated by only 5 neighbors versus 6 in the bulk. Nevertheless, the surface is neutral (and not very polar). This structure was supported by bond valence (BV) theory calculations. While BV theory is essentially an empirical methodology. It has been shown to produce remarkably accurate predictions for many materials applications [60].

Full AIMD simulations of 100 goethite + water interface (Surface I cleavage) were carried out. Solvating the slabs with $53 \mathrm{H}_{2} \mathrm{O}$ molecules led to a slight contraction of the distance to the nearest water from the surface (OII in Fig. 2). The average $\mathrm{Fe}-\mathrm{OH}_{2}$ bond distances in the AIMD simulations that were found to be 2.40 and $2.34 \AA$ for the PBE96 and PBE96 + Grimme2 theories respectively were still considerably larger than $2.15 \AA$ distance seen in the model fitted from CTR data [22] and the 2.13-2.21 $\AA$ distances from prior MD calculations [27]. In summary all the DFT calculations support the long bond and weak interaction. The good agreement of the BV methodology with DFT predictions of bond length changes support the further investigation of this method for surface structures.

The polarization of the water layer due to the surface (and vice versa) is small and localized only to the immediate vicinity of the interface. Density difference plots and Wannier-Boys orbitals analysis support the classification into three types of water molecules observed in the simulations (see Fig. 7). The first is the capping water molecule (OII, Fig. 7) that is loosely bonded to the surface $\mathrm{Fe}^{3+}$. The second (OIII, Fig. 7) is hydrogen bonded to the surface hydroxyl, and the third type is similar to bulk water. The analysis shows that by the third layer the coordination and bonding are essentially that of bulk water. Even though the OII and OIII water molecules form ordered water layers on surface they do not bond to the surface strongly and as a result these water molecules readily exchanged with the bulk-like water molecules.

These results might suggest that other mineral surfaces may also have surface cations with only five neighbors. For instance, oxide surfaces where a surface cation is capped by a water molecule such as the (100) plane of diaspore, and the R-planes of sapphire and hematite.

\section{Abbreviations \\ AIMD: ab initio molecular dynamics; BO: bond order; CTR: crystal truncation rod; DFT: density functional theory; EXAFS: extended X-ray absorption fine structure; MD: molecular dynamics; MUSIC: multi site complexation; NWPW: NorthWest Plane-Wave module of NWChem; PBE96: Perdew, Burke and Ernzerhof 1996 exchange correlation functional; PBEO: hybrid extension of Perdew, Burke and Ernzerhof 1996 exchange correlation functional; LPO: Ione pair orbital; WBFC: Wannier-Boys Function Center.}

\section{Authors' contributions}

The manuscript was written and reviewed by all authors, YC and EB carried out the simulations and prepared all figures and tables. EB developed the AIMD simulation code and other analysis codes used in this work. All authors read and approved the final manuscript.

\section{Author details \\ 1 Department of Chemistry and Biochemistry, University of California, San Diego, La Jolla, CA 92093, USA. ${ }^{2}$ William R. Wiley Environmental Molecular Sciences Laboratory, Pacific Northwest National Laboratory, Richland, WA 99354, USA.}

\section{Competing interests \\ The authors declare that they have no competing interests.}

\section{Availability of data and materials}

Simulation data and analysis programs used in this work can be obtained from the Open Science Framework (https://osf.io/) from the following link: https://osfio/cz7d7/. The AIMD simulation program used in this work can be obtained from the NWChem website (http://www.nwchem-sw.org/). 


\section{Funding}

This material is based upon work supported by the U.S. Department of Energy, Office of Science, Office of Basic Energy Sciences, Chemical Sciences, Geosciences, and Biosciences Division DE-AC06-76RLO 1830. Additional support from EMSL operations. EMSL operations are supported by the DOE's Office of Biological and Environmental Research. We wish to thank the Scientific Computing Staff, Office of Energy Research, and the U. S. Department of Energy for a grant of computer time at the National Energy Research Scientific Computing Center (Berkeley, CA). Some of the calculations were performed on the Cascade computing systems at the Molecular Science Computing Facility in the William R. Wiley Environmental Molecular Sciences Laboratory (EMSL) at PNNL.

Received: 28 December 2016 Accepted: 10 March 2017

Published online: 31 March 2017

\section{References}

1. Brown GE et al (1999) Metal oxide surfaces and their interactions with aqueous solutions and microbial organisms. Chem Rev 99(1):77-174

2. Kubicki JD, Paul KW, Sparks DL (2008) Periodic density functional theory calculations of bulk and the (010) surface of goethite. Geochem Trans 9:4

3. Majzlan J, Gravel KD (2003) Thermodynamics of Fe of goethite (a-FeOOH), lepidocrocite ( $g$-FeOOF) and maghemite ( $g$-fe2O3). Am Miner 88:855-859

4. van der Zee C et al (2003) Nanogoethite is the dominant reactive oxyhydroxide phase in lake and marine sediments. Geology 31(11):993-996

5. Hochella MF et al (2008) Nanominerals, mineral nanoparticles, and Earth systems. Science 319(5870):1631-1635

6. Klingelhofer G et al (2005) Mossbauer spectroscopy on Mars: goethite in the Columbia Hills at Gusev crater. Hyperfine Interact 166(1-4):549-554

7. Villalobos M, Trotz MA, Leckie JO (2003) Variability in goethite surface site density: evidence from proton and carbonate sorption. J Colloid Interface Sci 268(2):273-287

8. Rustad JR, Felmy AR, Hay BP (1996) Molecular statics calculations of proton binding to goethite surfaces: a new approach to estimation of stability constants for multisite surface complexation models. Geochim Cosmochim Acta 60(9):1563-1576

9. Fendorf $S$ et al (1997) Arsenate and chromate retention mechanisms on goethite. 1. Surface structure. Environ Sci Technol 31(2):315-320

10. Boily J-F et al (2000) Benzenecarboxylate surface complexation at the goethite ( $\mathrm{a}-\mathrm{FeOOH}) /$ water interface: I. A mechanistic description of pyromellitate surface complexes from the combined evidence of infrared spectroscopy, potentiometry, adsorption data, and surface complexation modeling. Langmuir 16(13):5719-5729

11. van Geen A, Robertson AP, Leckie JO (1994) Complexation of carbonate species at the goethite surface: implications for adsorption of metal ions in natural waters. Geochim Cosmochim Acta 58(9):2073-2086

12. Watts HD, Tribe L, Kubicki JD (2014) Arsenic adsorption onto minerals: connecting experimental observations with density functional theory calculations. Minerals 4(2):208-240

13. Antelo J et al (2005) Effects of $\mathrm{pH}$ and ionic strength on the adsorption of phosphate and arsenate at the goethite-water interface. J Colloid Interface Sci 285(2):476-486

14. Cheng T et al (2004) Effects of phosphate on uranium (VI) adsorption to goethite-coated sand. Environ Sci Technol 38(22):6059-6065

15. Duff M, Amrhein C (1996) Uranium (VI) adsorption on goethite and soil in carbonate solutions. Soil Sci Soc Am J 60(5):1393-1400

16. Sanchez AL, Murray JW, Sibley TH (1985) The adsorption of plutonium IV and $V$ on goethite. Geochim Cosmochim Acta 49(11):2297-2307

17. Cockcroft J (1999) A hypertext book of crystallographic space group diagrams and tables. http://img.chem.ucl.ac.uk/sgp/mainmenu.htm

18. Dutch S (2011) Three-dimensional space groups. http://www.uwgb.edu/ dutchs/SYMMETRY/3dSpaceGrps/3dspgrp.htm

19. Buerger MJ (1963) Elementary crystallography

20. Rustad JR, Felmy AR, Hay BP (1996) Molecular statics calculations for iron oxide and oxyhydroxide minerals: toward a flexible model of the reactive mineral-water interface. Geochim Cosmochim Acta 60(9):1553-1562
21. Randall SR et al (1999) The mechanism of cadmium surface complexation on iron oxyhydroxide minerals. Geochim Cosmochim Acta 63(19-20):2971-2987

22. Ghose SK, Waychunas GA, Trainor TP, Eng PJ (2010) Hydrated geothite (a-FeOOH) (100) interface structure: ordered water and surface functional groups. Geochim Cosmochim Acta 74:1943-1953

23. Fenter P, Sturchio NC (2004) Mineral-water interfacial structures revealed by synchrotron X-ray scattering. Prog Surf Sci 77(5-8):171-258

24. Shroll RM, Straatsma TP (2003) Molecular dynamics simulations of the goethite-water interface. Mol Simul 29(1):1-11

25. Boily J-F (2012) Water structure and hydrogen bonding at goethite/ water interfaces: implications for proton affinities. J Phys Chem C 116(7):4714-4724

26. Rustad JR, Felmy AR, Bylaska EJ (2003) Molecular simulation of the magnetite-water interface. Geochim Cosmochim Acta 67(5):1001-1016

27. de Leeuw NH, Cooper TG (2007) Surface simulation studies of the hydration of white rust $\mathrm{Fe}(\mathrm{OH})(2)$, goethite alpha- $\mathrm{FeO}(\mathrm{OH})$ and hematite alphaFe(2)O(3). Geochim Cosmochim Acta 71(7):1655-1673

28. Boily JF (2012) Water structure and hydrogen bonding at goethite/ water interfaces: implications for proton affinities. J Phys Chem C 116(7):4714-4724

29. Kerisit S (2011) Water structure at hematite-water interfaces. Geochim Cosmochim Acta 75(8):2043-2061

30. Kohanoff J (1994) Phonon spectra from short non-thermally equilibrated molecular dynamics simulations. Comput Mater Sci 2(2):221-232

31. Kerisit S, Rosso KM (2006) Computer simulation of electron transfer at hematite surfaces. Geochim Cosmochim Acta 70(8):1888-1903

32. Fenter $P$ et al (2013) Is the calcite-water interface understood? Direct comparisons of molecular dynamics simulations with specular X-ray reflectivity data. J Phys Chem C 117(10):5028-5042

33. Kerisit S, Ilton ES, Parker SC (2006) Molecular dynamics simulations of electrolyte solutions at the (100) goethite surface. J Phys Chem B 110(41):20491-20501

34. Hiemstra T, VanRiemsdijk WH (1996) A surface structural approach to ion adsorption: the charge distribution (CD) model. J Colloid Interface Sci 179(2):488-508

35. Fitts JP et al (2005) Second-harmonic generation and theoretical studies of protonation at the water/alpha- $\mathrm{TiO}_{2}$ (110) interface. Chem Phys Lett 411(4-6):399-403

36. Peacock $C L$, Sherman DM (2004) Vanadium(V) adsorption onto goethite (alpha-FeOOH) at pH 1.5 to 12: a surface complexation model based on ab initio molecular geometries and EXAFS spectroscopy. Geochim Cosmochim Acta 68(8):1723-1733

37. Zhou L, Xiu F, Qiu M, Xia S, Yu L (2017) The adsorption and disociation of water molecule on geothite (010)surface: A DFT approach. Appl Surf Sci 392:760-767

38. Becke AD (2014) Perspective: fifty years of density-functional theory in chemical physics. J Chem Phys 140(18):18A301

39. Brown ID (1987) Recent developments in the bond valence model of inorganic bonding. Phys Chem Miner 15(1):30-34

40. Car R, Parrinello M (1985) Unified approach for molecular-dynamics and density-functional theory. Phys Rev Lett 55(22):2471-2474

41. Marx D, Hutter J (2012) Ab initio molecular dynamics: basic theory and advanced methods. Cambridge University Press, Cambridge

42. Bylaska E et al. (2011) Large-scale plane-wave-based density functional theory: formalism, parallelization, and applications. In: Computational methods for large systems: electronic structure approaches for biotechnology and nanotechnology, pp 77-116

43. Valiev M, Bylaska EJ, Govind N, Kowalski K, Straatsma TP, Van Dam HJ, Wang D, Nieplocha J, Apra E, Windus TL, De Jong WA (2010) NWChem: a comprehensive and scalable open-source solution for large scale molecular simulations. Comput Phys Commun 181(9):1477-1489

44. Perdew JP, Burke K, Ernzerhof M (1997) Generalized gradient approximation made simple (vol 77, pg 3865, 1996). Phys Rev Lett 78(7):1396

45. Grimme S et al (2010) A consistent and accurate ab initio parametrization of density functional dispersion correction (DFT-D) for the 94 elements H-Pu. J Chem Phys 132(15):154104

46. Gillan MJ, Alfe D, Michaelides A (2016) Perspective: how good is DFT for water? J Chem Phys 144(13):130901 
47. Adamo C, Barone V (1999) Toward reliable density functional methods without adjustable parameters: the PBEO model. J Chem Phys 110(13):6158-6170

48. Bogatko S et al (2013) The aqueous $\mathrm{Ca}^{2+}$ system, in comparison with $\mathrm{Zn}^{2+}, \mathrm{Fe}^{3+}$, and $\mathrm{Al}^{3+}$ : an ab initio molecular dynamics study. Chem Eur J 19(9):3047-3060

49. Hamann DR (1989) Generalized norm-conserving pseudopotentials. Phys Rev B 40(5):2980-2987

50. Hamann DR, Schluter M, Chiang C (1979) Norm-conserving pseudopotentials. Phys Rev Lett 43(20):1494-1497

51. Troullier N, Martins JL (1991) Efficient pseudopotentials for plane-wave calculations. Phys Rev B 43(3):1993-2006

52. Bylander DM, Kleinman $L$ (1984) Outer-core electron and valence electron pseudopotential. Phys Rev B 29(4):2274-2276

53. Rochester $\mathrm{CH}$, Topham SA (1979) Infrared study of surface hydroxylgroups on goethite. J Chem Soc Faraday Trans I(75):591-602

54. Ying C, Bylaska E, Weare J (2016) Chapter 4: 1st principle estimation of geochemically important transition metal oxide properties: structure and dynamics of the bulk, surface and mineral/aqueous fluid interface. In: Kubicki J (ed) Molecular modeling of geochemical reactions: an introduction. Wiley, New York

55. Rosso KM, Rustad JR (2001) Structures and energies of $\mathrm{AlOOH}$ and $\mathrm{FeOOH}$ polymorphs from plane wave pseudopotential calculations. Am Miner 86(3):312-317

56. Tunega D (2012) Theoretical study of properties of goethite $(\mathrm{a}-\mathrm{FeOOH})$ at ambient and high-pressure conditions. J Phys Chem C 116(11):6703-6713

57. Nose S (1984) A unified formulation of the constant temperature molecular-dynamics methods. J Chem Phys 81(1):511-519

58. Hoover WG (1985) Canonical dynamics-equilibrium phase-space distributions. Phys Rev A 31(3):1695-1697

59. Blochl PE, Parrinello M (1992) Adiabaticity in 1st-principles moleculardynamics. Phys Rev B 45(16):9413-9416

60. Brown ID, Altermatt D (1985) Bond-valence parameters obtained from a systematic analysis of the inorganic crystal-structure database. Acta Crystallogr Sect B Struct Sci 41:244-247
61. Donnay $\mathrm{G}$, Allman $\mathrm{R}$ (1970) How to recognize $\mathrm{O}^{2-}, \mathrm{OH}^{-}$, and $\mathrm{H}_{2} \mathrm{O}$ in crystal structures determined by X-rays. Am Mineral 55(5-6):1003

62. Brown ID (2009) Recent developments in the methods and applications of the bond valence model. Chem Rev 109(12):6858-6919

63. Pauling $L$ (1929) The principles determining the structure of complex ionic crystals. J Am Chem Soc 51(1-4):1010-1026

64. Bergerhoff $\mathrm{G}$ et al (1983) The inorganic crystal-structure data-base. J Chem Inf Comput Sci 23(2):66-69

65. Lightstone FC et al (2005) A first-principles molecular dynamics study of calcium in water. ChemPhysChem 6(9):1745-1749

66. Bogatko SA, Bylaska EJ, Weare JH (2010) First principles simulation of the bonding, vibrational, and electronic properties of the hydration shells of the high-spin $\mathrm{Fe}^{3+}$ ion in aqueous solutions. J Phys Chem A 114(5):2189-2200

67. Kumar $\mathrm{N}$ et al (2011) Faster proton transfer dynamics of water on $\mathrm{SnO}_{2}$ compared to $\mathrm{TiO}_{2}$. J Chem Phys 134(4):044706

68. Bogatko $\mathrm{S}$ et al (2013) The aqueous $\mathrm{Ca}^{2+}$ system, in comparison with $\mathrm{Zn}^{2+}, \mathrm{Fe}^{3+}$, and $\mathrm{Al}^{3+}$ : an ab initio molecular dynamics study. Chem Eur J 19(9):3047-3060

69. Bylaska EJ et al (2007) Structure and dynamics of the hydration shells of the $\mathrm{Al}^{3+}$ ion. J Chem Phys 126(10):104505

70. Richens DT (2005) Ligand substitution reactions at inorganic centers. Chem Rev 105(6):1961-2002

71. Langford CH, Gray HB (1965) Ligand substitution processes. W.A. Benjamin, New York

72. Nichols P et al (2008) Equatorial and apical solvent shells of the UO22+ ion. J Chem Phys 128(12):124507

73. Atta-Fynn R et al (2011) Hydration shell structure and dynamics of curium (III) in aqueous solution: first principles and empirical studies. J Phys Chem A 115(18):4665-4677

\section{Submit your manuscript to a SpringerOpen ${ }^{\circ}$ journal and benefit from:}

- Convenient online submission

Rigorous peer review

- Immediate publication on acceptance

- Open access: articles freely available online

- High visibility within the field

- Retaining the copyright to your article 